\title{
Angle-resolved 2D Imaging of Electron Emission Processes in Atoms and Molecules
}

\author{
E. Kukk*, , A.A. Wills* , B. Langer*, J.D. Bozek ${ }^{\dagger}$, and N. Berrah* \\ * Department of Physics, Western Michigan University, Kalamazoo, MI 49008-5151 \\ ${ }^{\dagger}$ Lawrence Berkeley National Laboratory, University of California, Berkeley, CA 94720
}

\begin{abstract}
A variety of electron emission processes have been studied in detail for both atomic and molecular systems, using a highly efficient experimental system comprising two time-of-flight (TOF) rotatable electron energy analyzers and a 3rd generation synchrotron light source. Two examples are used here to illustrate the obtained results. Firstly, electron emission in the $\mathrm{HCl}$ molecule have been mapped over a $14 \mathrm{eV}$ wide photon energy range over the $\mathrm{Cl} 2 \mathrm{p}$ ionization thresholds. Particular attention is paid to the dissociative core-excited states, for which the Auger electron emission shows photon energy dependent features. Also, the evolution of resonant Auger to the normal Auger decay distorted by post collision interaction has been observed and the resonating beahavior of the valence photoelectron lines studied. Secondly, an atomic system, neon, in which excitation of doubly excited states and their subsequent decay to various accessible ionic states has been studied. Since these processes only occurs via inter-electron correlations, the many body dynamics of an atom can be probed, revealing relativistic effects, surprising in such a light atom. Angular distribution of the decay of the resonances to the parity-unfavored continuum exhibits significant deviation from the LS coupling predicitons.
\end{abstract}

\section{INTRODUCTION}

The electron emission processes induced by VUV radiation have been studied extensively for relatively simple systems such as rare gas atoms and diatomic molecules. Electron spectroscopy of free atoms and molecules has proven to be a valuable tool for testing theoretical models of electronic structure, dynamics and of many-body effects. Rapid development of experimental systems and excitation sources also continues to reveal novel and often surprising effects and details in atomic and molecular electron spectra. The results presented here benefitted from the combined use of 3rd generation synchrotron radiation source (Advanced Light Source at Lawrence Berkeley National Laboratory), highly efficient electron timeof-flight analyzers capable of recording angularly resolved data, and the use of sophisticated two-dimensional (2D) data acquisition and analysis software [1]. The 
2D imaging technique - electron emission intensity recorded as a function of the energies of both the exciting photons and emitted electrons - has proven to be an excellent tool for studying processes that are spred over broad ranges of excitation energies and/or kinetic energies of the emitted electrons. Two examples of such processes will be discussed below: (i) Auger decay of $2 p$ core excitations in the $\mathrm{HCl}$ molecule, especially in competition with molecular dissociation and (ii) excitation of doubly excited states in neon and their subsequent decay to various ionic states, revealing breakdown of LS coupling.

\section{EXPERIMENT}

The electron time-of-flight (TOF) spectra were measured at beamline 9.0.1 of the Advanced Light Source synchrotron radiation facility, which uses radiation from a 8$\mathrm{cm}$ 55-period undulator, monochromatized by a spherical grating monochromator. Two TOF analyzers [2] were mounted in a plane perpendicular to the photon beam propagation direction and were housed in a chamber rotatable around the photon beam (see Fig. 1). This setup allowed angle-resolved (with respect to the electric vector of the light) measurements simultaneously at two angles, $125.3^{\circ}$ apart, and at a different pair of angles after rotating the chamber.

Each TOF analyzer consists of a $678-\mathrm{mm}$ long drift tube with a pair of microchannel plates and a detector plate mounted at the end. To measure flight times of the electrons in the drift tube, the synchrotron source operated in a 'double-bunch'

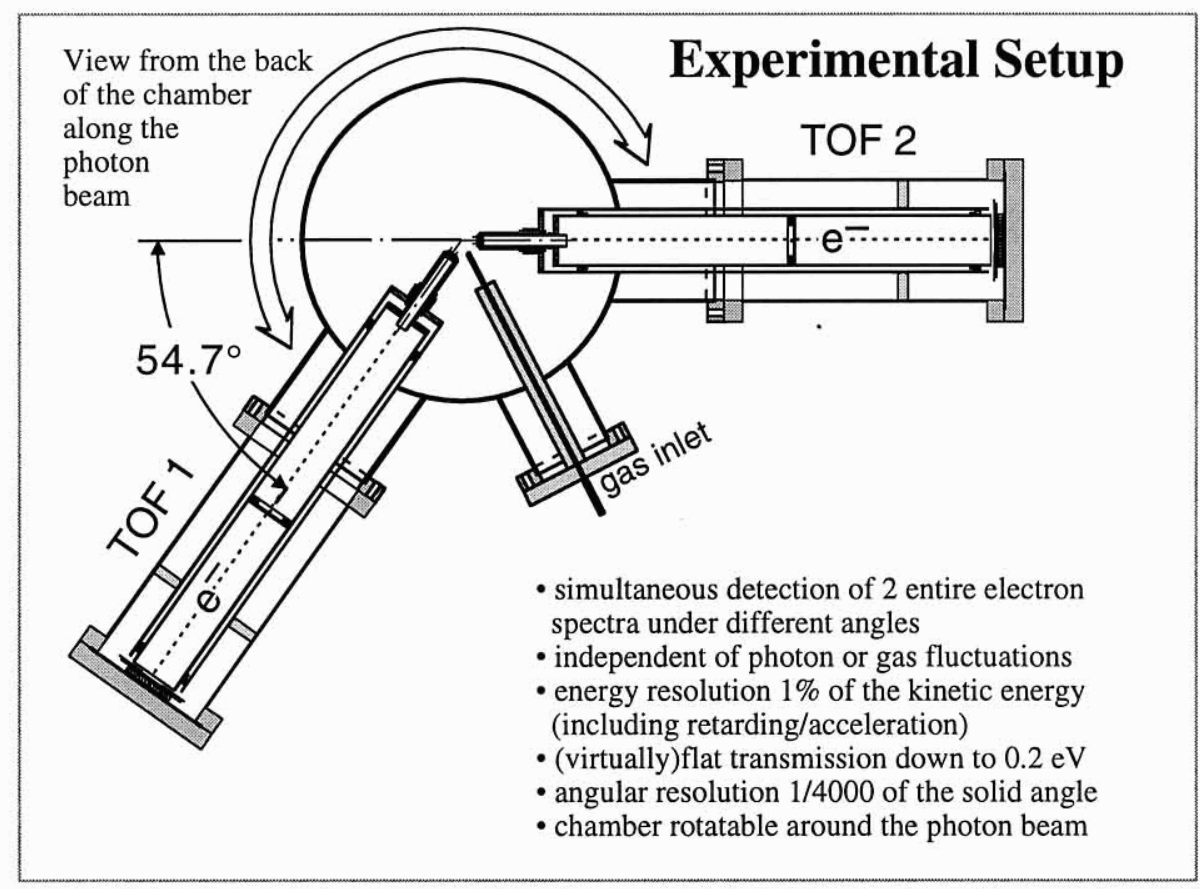

FIGURE 1. Schematic diagram of the experimental setup. 
mode, delivering short bursts of photons in $328 \mathrm{~ns}$ intervals, during which the emitted electrons had time to reach the detectors. In order to increase the flight times and thus improve the energy resolution, the electrons can be retarded prior to entering the field-free drift tube. The time scale of the original spectra was then converted to the kinetic energy scale using a procedure described in Ref. [3].

\section{AUGER DECAY OF CORE-EXCITED STATES IN THE HCL MOLECULE}

\section{Overview}

There have been numerous studies of inner shell processes in small molecules during recent years (e.g. Refs. [4-11]. These studies have, however, been performed at only a few selected photon energies. The results presented here give a more complete picture, since the electron emission spectra from $\mathrm{HCl}$ following photoexcitation/ionization of the $\mathrm{Cl} 2 p$ core levels were investigated by recording angle-resolved 2D data sets over a $14 \mathrm{eV}$ wide photon energy range in a kinetic energy window covering the majority of the ejected electrons. The resulting $2 \mathrm{D}$ images illustrate the evolution of the electron emission processes as the photon energy is scanned towards and across the $\mathrm{Cl} 2 p$ ionization thresholds.

The absorption spectrum of $\mathrm{HCl}$ around the $\mathrm{Cl} 2 p$ ionization threshold [12] can be divided into three regions: i) excitation to the antibonding $\sigma^{*}$ orbital $\left(2 p \rightarrow \sigma^{*}\right)$, ii) excitation to the Rydberg orbitals $(2 p \rightarrow n l)$ and iii) ionization into the continuum $(2 p \rightarrow \epsilon l)$. These three regions are indicated on the total electron yield curve along the right side of Fig. 2. The 2D electron emission map, shown in the main panel of Fig. 2, was obtained at the angle of $90^{\circ}$ relative to the polarization plane. In this map, intensity of the electron signal is represented as different shades of gray. Electrons emitted with the same kinetic energy at different photon energies are aligned vertically in the 2D map. Those emitted with constant binding energy form diagonal lines across the map. The features in the different regions of the 2D map are discussed in more detail below.

\section{Auger decay of dissociating states}

Broad bands in the total electron yield between 199.0 and $203.5 \mathrm{eV}$ correspond to excitation of the $2 p$ electrons to the first unoccupied molecular orbital, $\sigma^{*}$. When occupied, this antibonding orbital causes the core-excited molecule to dissociate. The dissociation,

$H C l^{*}\left(2 p^{5} \sigma^{*}\right) \rightarrow H(1 s)+C l^{*}\left(2 p^{5} 3 p^{6}\right)$,

produces neutral hydrogen and core-excited chlorine atoms and proceeds on a time scale $\left(\approx 10^{-14} \mathrm{~s}\right)$ that competes with the Auger decay of the core hole $[13,14]$. The light hydrogen atom moves quickly away from the $\mathrm{Cl}$ atom, so that a large 


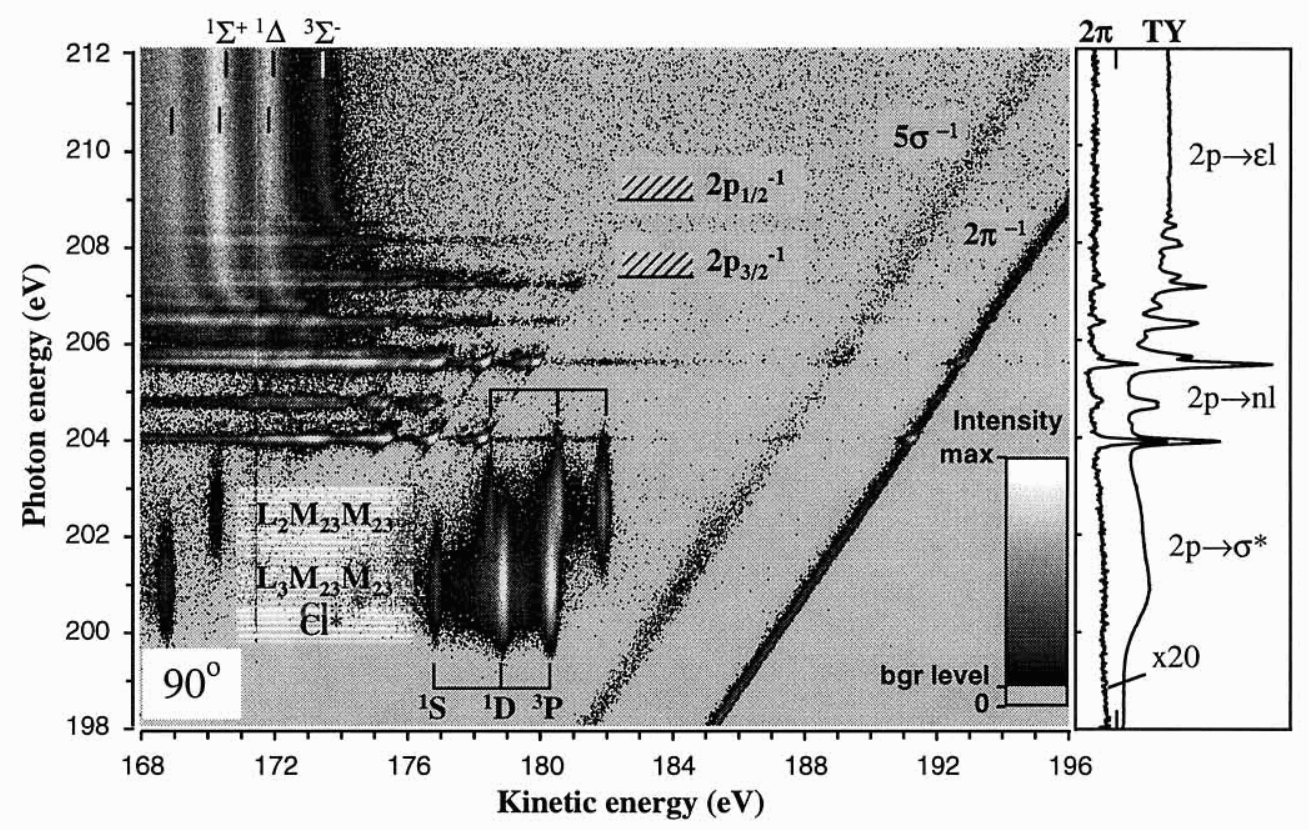

FIGURE 2. Two-dimensional (2D) map of electron emission from the $\mathrm{HCl}$ molecule across the $\mathrm{Cl} 2 p$ ionization threshold, taken at $90^{\circ}$ relative to the polarization plane. The total electron and partial $2 \pi$ photoelectron yields (marked as TY ans $2 \pi$, respectively) are shown on the right.

part of the Auger decay occurs in the atomic chlorine as $2 p^{5} 3 p^{6} \rightarrow 3 p^{4}$ transitions, forming intense vertical lines in the 2D map at the photon energies of the $2 p \rightarrow \sigma^{*}$ excitation, as can be seen in Fig. 2 and in more detail in Fig. 3. These sharp atomic lines have pronounced tails at their low kinetic energy side arising from Auger transitions in the molecular environment prior to the complete dissociation, which form a 'molecular background' to the atomic Auger spectrum [14].

As the photon energy is increased across the breadth of the $\sigma^{*}$ band, $\mathrm{HCl}$ molecules with decreasing internuclear separation are selected due to the projection of the ground state population onto the dissociative potential energy curve. This shift may influence the time scale of the early stage of the dissociation, which should be reflected in the balance between the molecular background and the sharp atomic peaks in the Auger spectra. In order to investigate this possibility, the intensity of one atomic line and its associated background can be monitored in the $2 \mathrm{D}$ maps as a function of photon energy. The $2 p_{1 / 2}^{-1} 3 p^{6} \rightarrow 3 p^{4}\left({ }^{3} P\right)$ atomic line at $182.0-\mathrm{eV}$ kinetic energy was selected, since it is well separated from neighboring peaks. The molecular tail of this line is assigned to transitions to the ${ }^{4} \Sigma^{-},{ }^{2} \Sigma^{-}$and ${ }^{4} \Pi$ states of $\mathrm{HCl}^{+}$[14]. The distinction between the atomic (marked 'A' in Fig. 3 and molecular ('M') regions is somewhat arbitrary. Here, we represent the latter by choosing the part of the spectrum between the ${ }^{1} D$ and ${ }^{3} P$ lines, from 180.8 to $181.8 \mathrm{eV}$. The comparison of the two regions shows that the molecular part of 


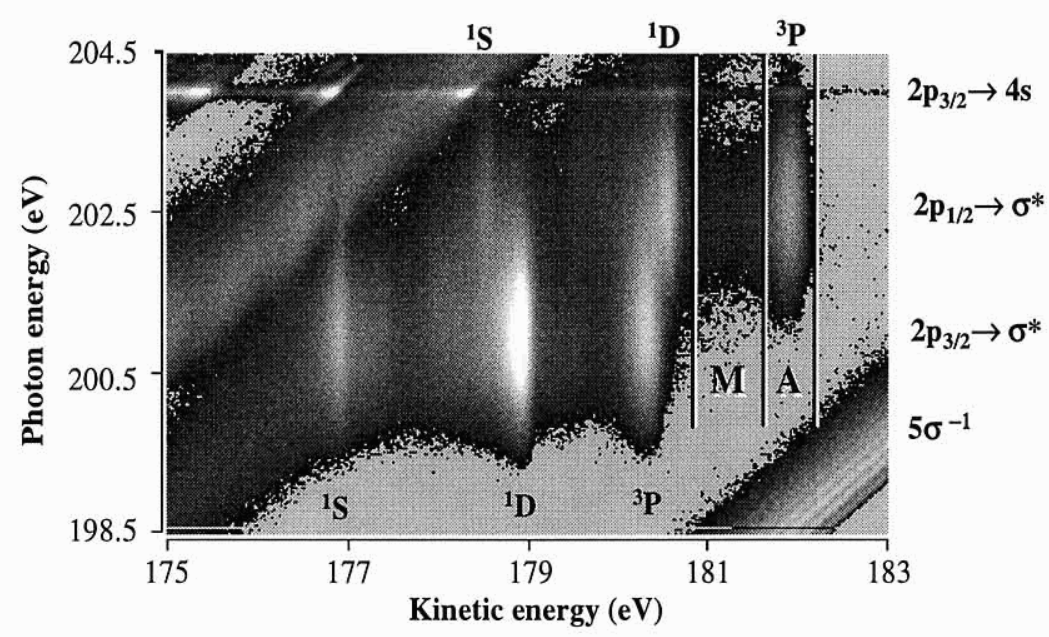

FIGURE 3. 2D map of Auger electron emission at the $2 p_{1 / 2,3 / 2}^{-1} \sigma^{*}$ resonances, taken at $35^{\circ}$.

the Auger spectrum dominates at photon energies well below the absorption peak maximum. With increasing photon energy, the internuclear distance at which the excitation can take place shifts to smaller values, where the energy of the core excited state is higher. Here the atomic peak gains intensity over the molecular background, inferring a higher dissociation rate. This effect can be related to the slope of the potential energy curve of the excited state, as discussed in Ref. [15].

Another novel effect connected to the Auger decay of the $\sigma^{*}$ resonaces can be observed in Fig. 3. The tail of the $2 p_{1 / 2}^{-1} \sigma^{*}$ resonance overlaps with the first Rydberg resonance, $2 p_{3 / 2}^{-1} 4 s$, at the $204.0 \mathrm{eV}$ photon energy. The intensity of the Auger lines from the decay of the dissociating $2 p_{1 / 2}^{-1} \sigma^{*}$ state shows a sharp increase at the $2 p_{3 / 2}^{-1} 4 s$ resonance. At the magic angle $\left(54.7^{\circ}\right)$, these Auger lines account for about $6 \%$ of the total Auger intensity at this photon energy. About half of their intensity comes from the overlap with the Gaussian-shaped tail of the $2 p_{1 / 2}^{-1} \sigma^{*}$ resonance. The remainder is a resonant enhancement indicating the possibility of predissociation on the first Rydberg resonance. The transition from the bound $2 p_{3 / 2}^{-1} 4 s$ to dissociative $2 p_{1 / 2}^{-1} \sigma^{*}$ potential energy curve must involve some interaction between the two spinorbit components of the $2 p$ orbital.

\section{Auger decay of Rydberg resonances and core-ionized states}

A quite different pattern of Auger decay can be seen in the region of the sharp core-to-Rydberg excitations starting at the $204.0 \mathrm{eV}$ photon energy. The spectral structures here arise from molecular Auger decay and are assigned within the simplest spectator model as the $2 p^{-1} n l \rightarrow(4 \sigma 5 \sigma 2 \pi)^{-2} n^{\prime} l^{\prime}$ transitions, where $n l$ and $n^{\prime} l^{\prime}$ 
describe the Rydberg electron in the Auger initial and final state, respectively [16]. The intensity of the Auger spectra of the higher Rydberg states gradually shifts towards lower kinetic energies with the increasing photon energy. This is due to the weakening of the influence of the spectator electron and higher shake-up probability. Finally, the $2 \pi^{-2} n^{\prime} l^{\prime}$ resonant Auger lines merge into the $2 \pi^{-2}\left({ }^{3} \Sigma^{-},{ }_{1}^{1} \Delta,{ }^{1} \Sigma^{+}\right)$ normal Auger lines. There is no sudden change in the Auger decay pattern at the $2 p$ ionization thresholds (marked in Fig. 2, from Ref. [12]). Rather there is a smooth evolution from the spectator to normal Auger spectra through a strong post-collision interaction (PCI). The energy shift of the normal Auger lines, caused by the PCI between the outgoing slow $2 p$ photoelectron and Auger electron, can be seen through threshold and down to the Rydberg states. The shift below the threshold is caused by the screening effect of the bound, core-excited electron.

\section{Anisotropy of the electron emission}

Within the dipole approximation, the differential cross-section of the electron emission at an angle $\theta$ with respect to the polarization plane is given by

$$
\frac{d \sigma_{i \rightarrow f}(\theta)}{d \Omega}=\frac{\sigma_{i \rightarrow f}^{T}}{4 \pi}\left(1+\beta P_{2}(\cos \theta)\right)
$$

where $\sigma_{i \rightarrow f}^{T}$ is the total cross-section, $\Omega$ is the solid angle and $P_{2}(\cos \theta)$ is the secondorder Legendre polynomial. The anisotropy parameter $\beta$ id derived from the shape of the initial and final state wavefunctions of the photoelectron in the case of direct ionization. The $\beta$-parameter for the Auger electrons is given as a product of a molecular alignment parameter $\beta_{m}$ and an intrinsic anisotropy parameter $c_{a}$ for the Auger decay [17]:

$$
\beta=\beta_{m} c_{a}
$$

2D maps similar to Fig. 2 were also measured at $0,35.3$ and 54.7 degrees. From these data sets the normalized intensities and $\beta$-parameters of the $2 \pi$ and $5 \sigma$ photoelectron lines can be followed continuously as a function of photon energy. Fig. 4 shows the results for the $2 \pi$ photoelectron line. Below the Rydberg resonances, it has an almost constant $\beta$-value of 1.6 , but it drops to 0.90 at the first Rydberg resonance. However, $\beta$ of the $5 \sigma$ line shows no such pronounced change. At the next strong (overlapping) resonances $2 p_{3 / 2}^{-1} 3 d$ and $2 p_{1 / 2}^{-1} 4 s$, the value of $\beta$ for the $2 \pi$ line drops again to 1.15. These changes in the value of $\beta$ indicate that a resonant valence ionization channel becomes available. This is consistent with the sudden increase in the photoelectron yield at these Rydberg resonances, probably due to participator Auger transitions to these ionic states. Such an effect at Rydberg resonances has not been observed for other diatomic molecules like CO [11].

The $\beta$-values of the individual Auger lines display large variations (between -0.7 and 0.7 ) in the spectra measured at the $2 p^{-1} \sigma^{*}$ resonances, but show a markedly 


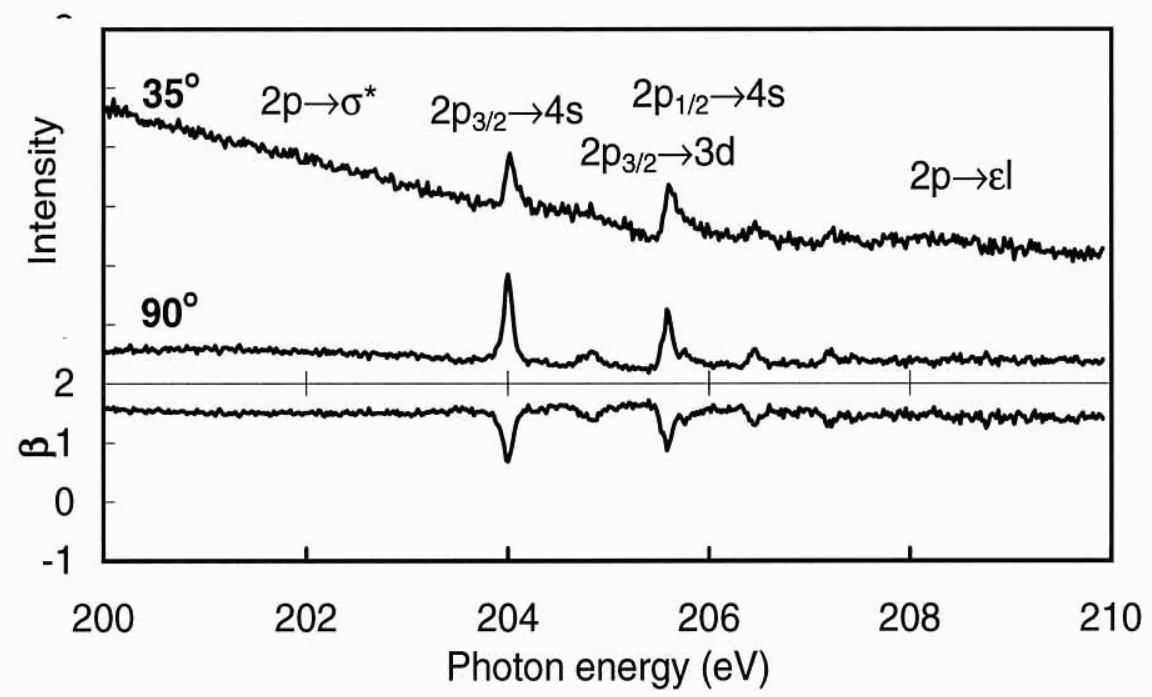

FIGURE 4. $2 \pi$ photoelectron yield at different angles (upper panel) and the anisotropy parameter (lower panel) as a function of photon energy.

more uniform behavior at the Rydberg resonances. At the $2 p_{3 / 2}^{-1} 4 s$ resonance, all Auger lines have negative $\beta$-values. The $2 \pi^{-2}\left({ }^{1} \Delta\right) 4 s$ line at $E_{b}=27.3 \mathrm{eV}$ has the largest anisotropy with $\beta=-0.7(2)$, which infers that the molecule is strongly aligned after the $2 p_{3 / 2} \rightarrow 4 s$ excitation. We also observed negative $\beta$-values at the overlapping $2 p_{3 / 2}^{-1} 3 d, 2 p_{1 / 2}^{-1} 4 s$ resonances. All of the molecular Auger lines have similar $\beta$-values and for the the normal Auger lines, $\beta$-parameters are equal within the error limits, since there is no change in the branching ratios of the Auger lines at different angles.

\section{SPIN-ORBIT EFFECTS IN PARITY UNFAVORED PHOTOIONIZATION OF NEON}

The purpose of this study was to use high-precision measurements in order to reveal details in the dynamics of doubly-excited states. We were able to demonstrate that for certain resonances, the angular distribution of photoelectrons and the ratio of partial cross sections to individual fine structure levels both exhibit behavior that deviates markedly from LS coupling predictions. These observations provide further evidence for the breakdown of LS coupling. Particularly interesting from this viewpoint are the $2 p^{4} 3 p n s$, nd doubly-excited resonances in the photoionization-excitation to the $2 p^{4}\left({ }^{3} P\right) 3 s\left({ }^{2} P\right) \mathrm{Ne}^{+}$ionic state. These were examined using high-resolution 2D imaging of photoionization cross sections that are differential in photon energy, photoelectron energy, and photoemission angle.

The $2 p^{4} 3 p n s, n d$ resonances are particularly strong because the $\sim 10 \%$ mixing of the $2 p^{5} 3 p$ configuration in the $2 p^{6}$ ground state leads to the one-electron photoexci- 
tation $2 p^{5} 3 p \rightarrow 2 p^{4} 3 p n s, n d$ [18]. Breakdown of LS coupling for this light system is due to the spin-orbit interaction, which most likely causes mixing between certain LS-allowed $2 p^{4} 3 p n s, n d\left({ }^{1} P_{1}\right)$ resonances and their ${ }^{3} S_{1},{ }^{3} P_{1}$, and ${ }^{3} D_{1}$ LS-forbidden counterparts. Angle-resolved and level-resolved measurements have provided an insight into such spin-orbit effects.

Fig. 5 displays the photoelectron spectra at two angles ( 0 and 54.7 degrees). For this measurement, the photon energy resolution was set to $20 \mathrm{meV}$, which

FIGURE 5. Photoelectron yield as a function of photon energy and binding energy at $0^{\circ}$ and $54.7^{\circ}$. The upper graphs show the spectrum at $51.3 \mathrm{eV}$ photon energy, and the vertical bars indicate the positions of the a) $2 s 2 p^{6}\left({ }^{2} S\right)$, b) $2 s^{2} 2 p^{4}\left({ }^{3} P\right) 3 s\left({ }^{4} P\right)$, and c) $2 s^{2} 2 p^{4}\left({ }^{3} P\right) 3 s\left({ }^{2} P\right)$ fine-structure levels. 
is a significant improvement over the previous resolution of $100 \mathrm{meV}$ in earlier differential measurements [19].

\section{LS-forbidden features in the angular distribution of the $2 p^{4} 3 s\left({ }^{2} P,{ }^{4} P\right)$ photoionization satellites}

A useful way of viewing the underlying dynamics of the differential cross-section (Eq. 1) is to consider the angular momentum transfer $\vec{j}_{t}$ [20]. The differential cross section reduces to an incoherent sum of terms associated with the allowed values of $j_{t}$, which are $\ell-1, \ell$ and $\ell+1$. While the parity-favored contributions $j_{t}=\ell \pm 1$ have complicated, energy-dependent angular distributions in general, the partial differential cross sections for all parity unfavored transitions $j_{t}=\ell$ have the analytic property $\left(\frac{d \sigma}{d \Omega}\right)_{j_{t}=\ell} \sim\left(\frac{d \sigma}{d \Omega}\right)_{j_{t}=\ell} \propto \sin ^{2} \theta$ independent of energy, or equivalently, $\beta_{\text {unf }}=-1$ [21]. One important consequence is that the parityunfavored contribution to the differential cross section vanishes at $\theta=0^{\circ}$.

For photoionization from the $2 p^{6}\left({ }^{1} S\right)$ ground state to the $2 p^{4} 3 s\left({ }^{2} P\right) \epsilon p\left({ }^{1} P\right)$ continuum, the angular momentum transfer is restricted to a single, parity-unfavored value $j_{t}=\ell=1$. This means that, in LS coupling, this transition is completely parity unfavored and the differential cross section vanishes at $\theta=0^{\circ}$, or equivalently, detection of photoelectrons at $0^{\circ}$ is an unmistakable indication of spin-orbit effects.

It is easy to observe the above-mentioned LS-forbidden features in Fig. 5. Firstly, the vertical lines (b) at $48.8 \mathrm{eV}$ binding energy (corresponding to the $2 p^{4} 3 s\left({ }^{4} \mathrm{P}\right.$ ) satellite) show, on certain resonances, noticeable photoelectron yields at both angles. Secondly, by comparing the photoelectron yields at $54.7^{\circ}$ and $0^{\circ}$ along the vertical lines (c) (corresponding to the $2 p^{4} 3 s\left({ }^{2} P\right)$ satellite at $49.4 \mathrm{eV}$ ), it can be seen that several resonances appear at both angles while the others disappear in the $0^{\circ}$ spectrum. For instance, the $2 p^{4}\left({ }^{3} P\right) 3 p\left({ }^{2} P\right) 3 d$ resonance at (photon energy) 51.3 $\mathrm{eV}$ shows a photoelectron yield that is almost equally strong at $0^{\circ}$ as it is at $54.7^{\circ}$, whereas the $2 p^{4}\left({ }^{1} D\right) 3 s\left({ }^{2} D\right) 4 p$ resonance at $50.6 \mathrm{eV}$ disappears almost completely in the $0^{\circ}$ spectrum. Since this is a parity unfavored transition in LS coupling, the appearance of any signal at $0^{\circ}$ is an immediate indication of the breakdown of LS coupling. The appearance of only certain resonances in the photoelectron yield at $0^{\circ}$ suggests that spin-orbit mixing between singlet and triplet resonances, not between ${ }^{1} P_{1}$ continuum states, is responsible for these LS-forbidden observations.

\section{Deviations of the ratio of the $2 p^{4} 3 s\left({ }^{2} P_{3 / 2}\right)$ and $2 p^{4} 3 s\left({ }^{2} P_{1 / 2}\right)$ satellite lines from its LS coupling value}

The high resolution in photoelectron energy allows easy identification of one additional LS-forbidden feature. By observing the photoelectron intensity along the two vertical lines (c) in Fig. 5 corresponding to the $2 p^{4} 3 s\left({ }^{2} P_{3 / 2}\right)$ and $2 p^{4} 3 s\left({ }^{2} P_{1 / 2}\right)$ 
satellites (at $49.35 \mathrm{eV}$ and $49.42 \mathrm{eV}$ binding energies, respectively), it can clearly be seen that some resonances such as $2 p^{4}\left({ }^{1} D\right) 3 s\left({ }^{2} D\right) 4 p$ preferentially autoionize to the ${ }^{2} P_{3 / 2}$ ionic state, yielding a ratio $r>2$, while others such as $2 p^{4}\left({ }^{3} P\right) 3 p\left({ }^{2} P\right) 3 d$ preferentially autoionize to the ${ }^{2} P_{1 / 2}$ state, yielding a ratio $r<1$. As a more quantitative diagnostic, the photoelectron yield along each of the two fine-structuresplit vertical lines (c) in Fig. 5 can be summed to give partial cross sections to the $3 / 2$ and $1 / 2$ levels. These indicate clearly, which resonances exhibit LS-forbidden ratios $r \neq 2$. In particular, the $2 p^{4}\left({ }^{1} D\right) 3 s\left({ }^{2} D\right) 4 p$ and $2 p^{4}\left({ }^{3} P\right) 3 p\left({ }^{2} P\right) 3 d$ resonances show ratios of $r=4.3$ and $r=0.2$, respectively. This is additional evidence for the breakdown of LS-coupling.

\section{CONCLUSIONS}

High-resolution and high-brighteness VUV radiation, combined with high performance experimental systems, has allowed detailed studies of some aspects of the dynamics of a molecule, $\mathrm{HCl}$, and of an atom, Ne. We hope that our measurements will stimulate further theoretical work in these areas.

\section{ACKNOWLEDGEMENTS}

This work was supported by the US Department of Energy, Office of Basic Energy Science, Division of Chemical Science under contract No. DE-FG02-95ER14299.

\section{REFERENCES}

1. A.A. Wills, D. Cubrić, M. Ukai, F. Currell, B.J. Goodwin, T. Reddish, and J. Comer, J. Phys. B 26, 2601 (1993).

2. B. Langer, A. Farhat, B. Nessar, N. Berrah, O. Hemmers, and J.D. Bozek, in Proceedings of the Atomic Physics with Hard X-rays from High Brilliance Synchrotron Light Sources Workshop, ANL/APS/TM-16 (Argonne National Laboratory, Argonne, IL, 1996), p. 245; A. Farhat, M. Humphrey, B. Langer, N. Berrah, J.D. Bozek, D. Cubaynes, Phys. Rev. A 56, 501 (1997).

3. N. Berrah, B. Langer, J.D. Bozek, T.W. Gorczyca, O. Hemmers, D.W. Lindle, and O. Toader, J. Phys. B 29, 1 (1996).

4. U. Becker, R. Hölzel, H.G. Kerkhoff, B. Langer, D. Szostak, and R. Wehlitz, Phys. Rev. Lett. 56, 1455 (1986).

5. O. Hemmers, F. Heiser, J. Eiben, R. Wehlitz, and U. Becker, Phys. Rev. Lett. 71, 987 (1993).

6. U. Hergerhahn and U. Becker, J. Electron Spectrosc. 72, 243 (1995).

7. U. Becker and A. Menzel, Nucl. Instr. Meth. in Physics B 99, 68 (1995).

8. H. Aksela, E. Kukk, S. Aksela, O.-P. Sairanen, A. Kivimäki, E. Nõmmiste, A. Ausmees, S.J. Osborne, S. Svensson, J. Phys. B 28, 4259 (1995).

9. M. Neeb, M. Biermann, and W. Eberhardt, J. Electron Spectrosc. 69, 239 (1994). 
10. A. Kivimäki, M. Neeb, B. Kempgens, H.M. Köppe, A.M. Bradshaw, Phys. Rev. A 54, 2137 (1996).

11. S. Sundin, S.J. Osborne, A. Ausmees, O. Björneholm, S.L. Sorensen, A. Kikas, and S. Svensson, Phys. Rev. A 56, 480 (1997).

12. D.A. Shaw, D. Cvejanović, G.C. King, and F.D. Read, J. Phys. B 17, 1984 (1984).

13. P. Morin and I. Nenner, Phys. Rev. Lett. 56, 1913 (1986).

14. E. Kukk, H. Aksela, O.-P. Sairanen, S. Aksela, A. Kivimäki, E. Nõmmiste, A. Ausmees, A. Kikas, S.J. Osborne, and S. Svensson, J. Chem. Phys. 104, 4475 (1996).

15. E. Kukk, A. Wills, N. Berrah, B. Langer, J.D. Bozek, O. Nayadin, M. Alserhi, A. Farhat, and D. Cubaynes, Phys. Rev. A 57, R1485 (1998).

16. E. Kukk, H. Aksela, O.-P. Sairanen, E. Nõmmiste, S. Aksela, S.J. Osborne, A. Ausmees, and S. Svensson, Phys. Rev. A 54, 2121 (1996).

17. D. Dill, J.R. Swanson, S. Wallace, and J.L. Dehmer, Phys. Rev. Lett. 45, 1393 (1980).

18. K. Schulz et al., Phys. Rev. A 54, 3095 (1996).

19. A. A. Wills et al., J. Phys. B 23, 2013 (1990).

20. U. Fano and D. Dill, Phys. Rev. A 6, 185 (1972).

21. D. Dill and U. Fano, Phys. Rev. Lett. 29, 1203 (1972). 